Submitted to the Annals of Statistics

arXiv: math.PR/0000000

\title{
HALF-TREK CRITERION FOR GENERIC IDENTIFIABILITY OF LINEAR STRUCTURAL EQUATION MODELS
}

\author{
By Rina Foygel* ${ }^{*}$ Jan Draisma ${ }^{\dagger, \ddagger}$ And Mathias Drton* \\ The University of Chicago* and Eindhoven University of Technology ${ }^{\dagger}$ and \\ Centrum voor Wiskunde en Informatica ${ }^{\ddagger}$
}

In Section 1 of this supplementary article, we provide lists of some mixed graphs on $m=5$ nodes, that are either rationally identifiable or generically infinite-to-one, but that cannot be classified as such using the half-trek criterion. Sections 2 and 3 contain proofs of lemmas used in the main paper for proving the HTC-identifiability and HTC-infinite-to-one criteria. Sections 4 and 5 contain proofs of our results, stated in the main paper, that GCidentifiable graphs are also HTC-identifiable and that the HTC-criterion can be strengthened through graph decomposition. Section 6 gives correctness proofs for the polynomial-time algorithms in the main paper for checking the HTC-criteria, and Section 7 discusses the weak HTC-criteria. Finally, Section 8 discusses the algebraic and computational content of the polynomial equations in the main paper that led to the HTC-criteria.

1. HTC-inconclusive graphs on $\boldsymbol{m}=\mathbf{5}$ nodes. In Section 7 of the main paper we found that there are 27 acyclic graphs that are generically infinite-to-one but HTC-inconclusive. They are listed in the last column of Table 1. This table also lists 112 rationally identifiable acyclic graphs that are HTC-inconclusive. Although initially, 121 graphs of this type were found in Section 7, we exclude the 9 graphs from this set that were found to be HTC-identifiable after decomposition into bidirected connected components; compare Section 8 of the main paper. For the cyclic case, there are too many graphs in this "gap" to enumerate, but in Table 2 we list 75 graphs of each of these two types, chosen randomly from the complete lists.

Each graph is specified by a pair of numbers $(d, b)$, which identify the directed and bidirected parts of the graph, respectively. The following algorithm converts a pair $(d, b)$ to a graph $G$ on $m$ nodes:

1. For $v=1,2, \ldots, m$, for $w=1,2, \ldots, v-1, v+1, \ldots, m$,

AMS 2000 subject classifications: $62 \mathrm{H} 05,62 \mathrm{~J} 05$

Keywords and phrases: Covariance matrix, Gaussian distribution, graphical model, multivariate normal distribution, parameter identification, structural equation model 
Add edge $v \rightarrow w$ if $\bmod (d, 2)=1$; Replace $d \leftarrow\left\lfloor\frac{d}{2}\right\rfloor$.

2. For $v=1,2, \ldots, m-1$, for $w=v+1, \ldots, m$,

Add edge $v \leftrightarrow w$ if $\bmod (b, 2)=1$; Replace $b \leftarrow\left\lfloor\frac{b}{2}\right\rfloor$.

TABLE 1

All rationally identifiable or generically infinite-to-one acyclic mixed graphs on $m=5$ nodes that are HTC-inconclusive even after decomposition into bidirected connected components, specified as a pair $(d, b)$ identifying the directed and bidirected part (see text for explanation).

\begin{tabular}{|c|c|c|c|c||c|}
\hline \multicolumn{7}{|c|}{ Rationally identifiable } & Inf.-to-one \\
\hline$(45,15)$ & $(6275,188)$ & $(860,354)$ & $(5304,417)$ & $(841,914)$ & $(1167,15)$ \\
$(107,15)$ & $(1452,195)$ & $(5240,354)$ & $(348,449)$ & $(4680,914)$ & $(1182,57)$ \\
$(1099,15)$ & $(5832,195)$ & $(5480,354)$ & $(380,449)$ & $(329,915)$ & $(1129,79)$ \\
$(1182,58)$ & $(364,209)$ & $(60,369)$ & $(860,449)$ & $(840,915)$ & $(1182,113)$ \\
$(1197,58)$ & $(364,217)$ & $(300,369)$ & $(5480,449)$ & & $(1144,121)$ \\
$(45,79)$ & $(5064,226)$ & $(360,369)$ & $(329,466)$ & & $(5224,121)$ \\
$(1114,85)$ & $(5304,226)$ & $(60,373)$ & $(840,466)$ & & $(1178,185)$ \\
$(1182,86)$ & $(5544,226)$ & $(300,373)$ & $(4680,466)$ & & $(5224,217)$ \\
$(1146,92)$ & $(45,271)$ & $(841,387)$ & $(4696,466)$ & & $(1178,241)$ \\
$(60,113)$ & $(333,278)$ & $(845,393)$ & $(329,470)$ & & $(360,249)$ \\
$(300,113)$ & $(453,278)$ & $(4696,401)$ & $(4680,470)$ & & $(5513,402)$ \\
$(360,113)$ & $(461,278)$ & $(4936,401)$ & $(107,527)$ & & $(5513,404)$ \\
$(364,113)$ & $(845,284)$ & $(329,402)$ & $(5769,534)$ & & $(5257,406)$ \\
$(376,113)$ & $(492,291)$ & $(453,402)$ & $(4489,562)$ & & $(333,410)$ \\
$(484,113)$ & $(5320,291)$ & $(840,402)$ & $(5513,562)$ & & $(329,442)$ \\
$(1114,113)$ & $(5352,291)$ & $(841,402)$ & $(13704,562)$ & & $(840,442)$ \\
$(4456,113)$ & $(5832,291)$ & $(844,402)$ & $(360,625)$ & & $(4680,442)$ \\
$(5288,113)$ & $(45,303)$ & $(4680,402)$ & $(376,625)$ & & $(47,527)$ \\
$(360,115)$ & $(1452,305)$ & $(4696,402)$ & $(360,627)$ & & $(15,543)$ \\
$(360,117)$ & $(5288,305)$ & $(4936,402)$ & $(5257,658)$ & & $(45,543)$ \\
$(60,121)$ & $(5544,305)$ & $(5768,402)$ & $(5257,659)$ & & $(62,611)$ \\
$(300,121)$ & $(5513,308)$ & $(329,403)$ & $(4746,676)$ & & $(62,625)$ \\
$(4746,172)$ & $(13704,308)$ & $(840,403)$ & $(6275,676)$ & & $(316,625)$ \\
$(4747,172)$ & $(5288,309)$ & $(4680,403)$ & $(4993,802)$ & & $(316,626)$ \\
$(6275,172)$ & $(1114,341)$ & $(329,406)$ & $(841,899)$ & & $(30,627)$ \\
$(6307,172)$ & $(333,342)$ & $(4680,406)$ & $(329,914)$ & & $(300,627)$ \\
$(4746,188)$ & $(453,342)$ & $(453,410)$ & $(840,914)$ & & \\
\hline
\end{tabular}

2. Lemma on systems of half-treks of minimal total length, for determining HTC-identifiability. This lemma is needed for the proof of Theorem 1 in the main paper, which determines when graphs are HTCidentifiable.

Lemma 1. Suppose $Y, P \subset V$ are subsets of equal cardinality, and $\Pi$ : $Y \rightrightarrows P$ is a system of half-treks with no sided intersection, with minimal 
TABLE 2

Some rationally identifiable or generically infinite-to-one cyclic mixed graphs on $m=5$ nodes that are $H T C$-inconclusive, specified as a pair $(d, b)$ identifying the directed and bidirected part (see text for explanation).

\begin{tabular}{|c|c|c||c|c|c|}
\hline \multicolumn{2}{|c||}{ Rationally identifiable } & \multicolumn{3}{c|}{ Infinite-to-one } \\
\hline$(345,440)$ & $(74536,788)$ & $(75321,516)$ & $(6026,104)$ & $(75142,580)$ & $(4714,324)$ \\
$(5278,528)$ & $(6597,385)$ & $(75398,20)$ & $(5293,261)$ & $(70251,640)$ & $(70874,144)$ \\
$(71329,18)$ & $(4730,66)$ & $(70803,896)$ & $(5047,256)$ & $(1431,324)$ & $(69933,529)$ \\
$(81089,0)$ & $(5259,554)$ & $(5251,257)$ & $(39631,0)$ & $(4710,840)$ & $(70797,84)$ \\
$(4698,388)$ & $(5545,96)$ & $(4457,592)$ & $(6279,129)$ & $(75355,2)$ & $(92853,0)$ \\
$(455,13)$ & $(75112,72)$ & $(74883,522)$ & $(14440,393)$ & $(70219,672)$ & $(5770,84)$ \\
$(4714,41)$ & $(5275,516)$ & $(5545,530)$ & $(13677,64)$ & $(5293,386)$ & $(501,84)$ \\
$(70881,80)$ & $(74970,4)$ & $(5803,514)$ & $(6599,264)$ & $(74872,532)$ & $(70249,656)$ \\
$(4686,610)$ & $(4579,384)$ & $(350,112)$ & $(5547,384)$ & $(5798,776)$ & $(4971,257)$ \\
$(4998,608)$ & $(70594,65)$ & $(74883,2)$ & $(5523,336)$ & $(76918,8)$ & $(5029,776)$ \\
$(74963,512)$ & $(74921,66)$ & $(5065,152)$ & $(5058,612)$ & $(5813,256)$ & $(14444,8)$ \\
$(14456,194)$ & $(5321,153)$ & $(74950,260)$ & $(74931,129)$ & $(1113,296)$ & $(6339,276)$ \\
$(74886,268)$ & $(70474,640)$ & $(874,836)$ & $(5102,512)$ & $(61,578)$ & $(75434,16)$ \\
$(5058,304)$ & $(74922,66)$ & $(5563,512)$ & $(5287,592)$ & $(5291,4)$ & $(5533,80)$ \\
$(70821,513)$ & $(5515,290)$ & $(74890,38)$ & $(80500,64)$ & $(30876,640)$ & $(361,230)$ \\
$(74915,6)$ & $(4682,322)$ & $(13706,532)$ & $(70115,520)$ & $(5579,96)$ & $(92644,8)$ \\
$(301,300)$ & $(13160,65)$ & $(81076,0)$ & $(5094,260)$ & $(4810,610)$ & $(5539,66)$ \\
$(5267,82)$ & $(4938,448)$ & $(70851,32)$ & $(75379,256)$ & $(13736,192)$ & $(4517,450)$ \\
$(76852,128)$ & $(4457,520)$ & $(875,608)$ & $(74542,128)$ & $(4781,40)$ & $(71410,128)$ \\
$(70738,304)$ & $(4730,640)$ & $(1430,120)$ & $(77262,0)$ & $(75241,2)$ & $(14795,512)$ \\
$(5267,384)$ & $(14425,194)$ & $(361,214)$ & $(375,200)$ & $(70909,0)$ & $(287,800)$ \\
$(71075,516)$ & $(4730,514)$ & $(5499,512)$ & $(351,104)$ & $(1385,153)$ & $(5251,614)$ \\
$(4397,897)$ & $(13706,529)$ & $(4970,518)$ & $(71379,128)$ & $(5834,322)$ & $(293,34)$ \\
$(14441,10)$ & $(4665,641)$ & $(5251,418)$ & $(5861,256)$ & $(4714,568)$ & $(75577,256)$ \\
$(6629,512)$ & $(70358,1)$ & $(70179,296)$ & $(6341,64)$ & $(14440,129)$ & $(80227,128)$ \\
\hline
\end{tabular}


total length among all such systems. If for a system of treks $\Psi: Y \rightrightarrows P$ the monomial $\Psi(\lambda, \Omega)=\Pi(\lambda, \omega)$, then $\Psi=\Pi$.

Proof. Let $Y=\left\{y_{1}, \ldots, y_{n}\right\}, P=\left\{p_{1}, \ldots, p_{n}\right\}$, and $\Pi=\left\{\pi_{1}, \ldots, \pi_{n}\right\}$, where $\pi_{i}$ has source $y_{i}$ and target $p_{i}$. Since $\Pi$ has minimal total length among all systems of half-treks from $Y$ to $P$ with no sided intersection, $\Pi$ cannot have a sub-system of the form

$$
\left\{\begin{array}{l}
\pi_{i_{1}}: y_{i_{1}} \cdots y_{i_{2}} \cdots p_{i_{1}} \\
\pi_{i_{2}}: y_{i_{2}} \cdots y_{i_{3}} \cdots p_{i_{2}} \\
\vdots \\
\pi_{i_{r-1}}: y_{i_{r-1}} \cdots y_{i_{r}} \cdots p_{i_{r-1}} \\
\pi_{i_{r}}: y_{i_{r}} \cdots y_{i_{1}} \cdots p_{i_{r}}
\end{array}\right.
$$

If there were such a sub-system, each trek in the sub-system could be shortened, that is, replace $\pi_{i_{1}}: y_{i_{1}} \cdots y_{i_{2}} \cdots p_{i_{1}}$ with its second section, $y_{i_{2}} \cdots p_{i_{1}}$, etc. Therefore, we can relabel the elements of $Y, P$ and $\Pi$ such that $j \leq i$ if trek $\pi_{i}$ contains $y_{j}$.

Write the second system of treks as $\Psi=\left\{\psi_{1}, \ldots, \psi_{n}\right\}$, where $\psi_{i}$ has source $y_{i}$ and target $p_{\alpha(i)}$. Here, $\alpha$ is some permutation of the indices in $[n]$. We claim that $\alpha(n)=n$ and $\psi_{n}=\pi_{n}$. Assuming this is true, let $Y^{\prime}=\left\{y_{1}, \ldots, y_{n-1}\right\}$ and $P^{\prime}=\left\{p_{1}, \ldots, p_{n-1}\right\}$, and let $\Pi^{\prime}$ and $\Psi^{\prime}$ be the induced sub-systems of treks from $Y^{\prime}$ to $P^{\prime}$. The ordering on $Y^{\prime}$ follows the same rule as the ordering on $Y$. Then $\psi_{n}=\pi_{n}$ implies that $\Pi^{\prime}(\lambda, \omega)=\Pi(\lambda, \omega) / \pi_{n}(\lambda, \omega)=$ $\Psi(\lambda, \omega) / \psi_{n}(\lambda, \omega)=\Psi^{\prime}(\lambda, \omega)$. By induction on $n$, we conclude that $\Psi=\Pi$.

It remains to show that $\alpha(n)=n$ and $\psi_{n}=\pi_{n}$. Write

$$
\pi_{n}: y_{n} \circ \rightarrow z_{1}^{n} \rightarrow z_{2}^{n} \rightarrow \ldots \rightarrow z_{k}^{n}=p_{n},
$$

where $y_{n} \circ \rightarrow z_{1}^{n}$ represents that either $y_{n} \rightarrow z_{1}^{n}$ or $y_{n} \leftrightarrow z_{1}^{n}$. By definition of the ordering on $Y$, the node $y_{n}$ does not appear in any trek in $\Pi$, except for $\pi_{n}$. And, node $y_{n}$ appears only once in $\pi_{n}$, since $\Pi$ has minimal total length. Hence, the only edge in $\Pi$ containing $y_{n}$ is the edge $y_{n} \circ \rightarrow z_{1}^{n}$. Since $\Psi(\lambda, \omega)=\Pi(\lambda, \omega)$, this implies that the only edge in $\Psi$ containing $y_{n}$ is the same edge $y_{n} \circ \rightarrow z_{1}^{n}$. Therefore, $\psi_{n}$ must be of the form

$$
\psi_{n}: y_{n} \circ \rightarrow z_{1}^{n} \cdots \text {. }
$$

Case 1: The path $\psi_{n}$ consists of only the edge $y_{n} \circ \rightarrow z_{1}^{n}$. Then $z_{1}^{n} \in P$. If $z_{1}^{n}=p_{j}$ for $j<n$, then $\pi$ would have a sided intersection, which is a 
contradiction. Therefore, $z_{1}^{n}=p_{n}$. Since $\Pi$ is a system of minimal length, $\pi_{n}$ must also consist of only $y_{n} \circ \rightarrow z_{1}^{n}=p_{n}$, which show that $\psi_{n}=\pi_{n}$.

Case 2: The path $\psi_{n}$ is of the form

$$
\psi_{n}: y_{n} \circ \rightarrow z_{1}^{n} \rightarrow \ldots
$$

Since $\Pi$ has no sided intersection, there is no edge of the form $p_{n} \rightarrow$. in $\Pi$. Since $\Psi(\lambda, \Omega)=\Pi(\lambda, \omega)$, we obtain that $z_{1}^{n} \neq p_{n}$, and thus $k \geq 2$ in (2.1). Now observe that the only edge of the form $z_{1}^{n} \rightarrow \cdot$ in $\Pi$, is the edge $z_{1}^{n} \rightarrow z_{2}^{n}$; otherwise, two treks in $\Pi$ would have a sided intersection at $z_{1}^{n}$. It follows that

$$
\psi_{n}: y_{n} \circ \rightarrow z_{1}^{n} \rightarrow z_{2}^{n} \cdots .
$$

Continue now to add edges one at a time to the path $\psi_{n}$, applying the reasoning just used at all but the last edge of $\psi_{n}$. Reasoning as in Case 1 for the last edge of $\psi_{n}$, we find that $\psi_{n}$ and $\pi_{n}$ are both equal to

$$
y_{n} \circ \rightarrow z_{1}^{n} \rightarrow z_{2}^{n} \rightarrow \ldots \rightarrow z_{r}^{n}=p_{n} .
$$

This completes the proof that $\alpha(n)=n$ and $\psi_{n}=\pi_{n}$.

3. Jacobian lemma for determining HTC-infinite-to-one status. This lemma is needed for the proof of Theorem 2 in the main paper, which determines when graphs are HTC-infinite-to-one.

Lemma 2. Let $\mathbf{J}$ be the Jacobian for the set of equations

$$
\left[(I-\Lambda)^{T} \Sigma(I-\Lambda)\right]_{v w}=0 \quad \forall(v, w) \notin B \text { and } v \neq w
$$

which has entries

$$
\mathbf{J}_{\{v, w\},(u, v)}=-\left[(I-\Lambda)^{T} \Sigma\right]_{w u}, \quad\{v, w\} \in N, u \in \operatorname{pa}(v),
$$

where the indexing is over a nonsibling pair $\{v, w\} \in N$ (i.e. $(v, w) \notin B$ and $v \neq w)$ and a directed edge $(u, v) \in D$. If $\mathbf{J}$ does not have generically full column rank, then the parametrization $\phi_{G}$ is generically infinite-to-one.

Proof. The parametrization $\phi_{G}$ maps the $(|D|+|B|+m)$-dimensional set $\Theta=\mathbb{R}_{\text {reg }}^{D} \times P D(B)$ to the $\left(\begin{array}{c}m+1 \\ 2\end{array}\right)$-dimensional space of symmetric $m \times m$ matrices. Since $\phi_{G}$ is a rational map, its Jacobian matrix $J\left(\phi_{G}\right)$ achieves its maximal rank at generic points in $\Theta$. This maximal rank is the dimension of the image of $\phi_{G}$. If the dimension is smaller than $|D|+|B|+m$, then, for generic choices of $(\Lambda, \Omega) \in \Theta$, the fiber $\mathcal{F}(\Lambda, \Omega)$ has positive dimension and 
is, in particular, infinite. Therefore, our theorem is proven if we can show that, under the assumed conditions, the Jacobian of $\phi_{G}$ does not have a full column rank.

We now claim that the Jacobian of $\phi_{G}, J\left(\phi_{G}\right)$, is of full column rank at $(\Lambda, \Omega)$ if and only $\mathbf{J}$ has full column rank at $\Lambda$ when taking $\Sigma=\phi_{G}(\Lambda, \Omega)$.

Consider the two maps

$$
h:(\Lambda, \Omega) \mapsto\left(\Lambda, \phi_{G}(\Lambda, \Omega)\right) \quad \text { and } \quad g:(\Lambda, \Sigma) \mapsto(I-\Lambda)^{T} \Sigma(I-\Lambda),
$$

where the domain of $h$ is $\Theta$ and the domain of $g$ is $\mathbb{R}_{\mathrm{reg}}^{D} \times P D_{m}$. The composition of the two maps satisfies

$$
(g \circ h)(\Lambda, \Omega)=\Omega \text {. }
$$

Partition $J\left(\phi_{G}\right)=\left(J_{\Lambda}\left(\phi_{G}\right), J_{\Omega}\left(\phi_{G}\right)\right)$, where the two parts hold the partial derivatives with respect to the $|D|$ free entries of $\Lambda$ and the $|B|+m$ free entries of $\Omega$, respectively. Similarly, partition the Jacobian $J(g)=$ $\left(J_{\Lambda}(g), J_{\Sigma}(g)\right)$. Taking derivatives in (3.3), we obtain that

$$
J_{\Lambda}(g)\left(\Lambda, \phi_{G}(\Lambda, \Omega)\right)+J_{\Sigma}(g)\left(\Lambda, \phi_{G}(\Lambda, \Omega)\right) J_{\Lambda}\left(\phi_{G}\right)(\Lambda, \Omega)=0
$$

and

$$
J_{\Sigma}(g)\left(\Lambda, \phi_{G}(\Lambda, \Omega)\right) J_{\Omega}\left(\phi_{G}\right)(\Lambda, \Omega)=\left(\begin{array}{cc}
0 & 0 \\
0 & I
\end{array}\right)
$$

where we have ordered rows and columns such that the pairs $(v, w)$ defining elements in $N$ are listed first. Hence, the identity matrix in the lower-right block of the right-hand side of (3.5) is of size $|B|+m$, and indexed by $B \cup V$. Under the same ordering of rows, observe that using the Jacobian in (3.1) we have

$$
J_{\Lambda}(g)\left(\Lambda, \phi_{G}(\Lambda, \Omega)\right)=\left(\begin{array}{c}
\left.\mathbf{J}\right|_{\Sigma=\phi_{G}}(\Lambda, \Omega) \\
0
\end{array}\right) .
$$

Combining (3.4) and (3.5), we obtain

$$
J_{\Sigma}(g)\left(\Lambda, \phi_{G}(\Lambda, \Omega)\right) \cdot J\left(\phi_{G}\right)(\Lambda, \Omega)=\left(\begin{array}{ccc}
-\left.\mathbf{J}\right|_{\Sigma=\phi_{G}(\Lambda, \Omega)} & 0 & 0 \\
0 & I & 0
\end{array}\right)
$$

where the two blocks of rows are indexed by $N$ and $B \cup V$, and the three blocks of columns are indexed by $D, B \cup V$, and $N$. Now note that the restriction of $g$ obtained by fixing $\Lambda$ is an injective map with continuous inverse $\Sigma \mapsto(I-\Lambda)^{-T} \Sigma(I-\Lambda)^{-1}$. Therefore, the matrix $J_{\Sigma}(g)$ is invertible, and we deduce that the rank of $J\left(\phi_{G}\right)$ at $(\Lambda, \Omega)$ is equal to the sum of $|B|+m$ and the rank of $\mathbf{J}$ at $\Lambda$ and $\Sigma=\phi_{G}(\Lambda, \Omega)$. This proves our claim relating the rank of $J\left(\phi_{G}\right)$ and that of $\mathbf{J}$. 
4. Proofs for half-trek versus G-criterion. In this section, we assume that $G$ is an acyclic mixed graph whose vertex set $V=[m]$ is enumerated according to some topological ordering and that Theorem 4 from the main paper applies, making the graph GC-identifiable. Let $A_{v}$ be the sets from this theorem. Recalling Definition 6 from the main paper, for each node $v \in V$, let $Y_{v} \cup Z_{v}=A_{v}$ be the partition that, together with the systems of treks $\Pi_{v}: Y_{v} \rightrightarrows \mathrm{pa}(v)$ and $\Psi_{v}: Z_{v} \rightrightarrows S_{<}(v)$, witnesses that $A_{v}$ satisfies the G-criterion with respect to $v$. For each $v$, for each $\pi \in \Pi_{v}$ (and each $\psi \in \Psi_{v}$ ), define $\pi^{\prime}$ (or $\psi^{\prime}$ ) by extending $\pi$ (or $\psi$ ) with the edge $t(\pi) \rightarrow v$ (or $t(\psi) \leftrightarrow v)$, as in Definition 6 from the main paper.

Lemma 3. Consider any node $v \in V$. If $w \in \operatorname{Left}(\pi)$ for some trek $\pi \in \Pi_{v}$, then $w \neq v$ and $w \notin \operatorname{sib}(v)$.

Proof. Let $y$ and $t(\pi)$ be the source and the target of $\pi$, respectively.

First, suppose that $w<v$. If $w \in \operatorname{sib}(v)$, then there is a trek $\psi \in \Psi_{v}$ with source $z \in Z_{v}$ and target $w$ that extends to a trek $\psi^{\prime}$ when appending the edge $w \leftrightarrow v$ to the right-hand side. Since there is a sided intersection between $\psi^{\prime}$ and $\pi^{\prime}$, we cannot have $w \in S_{<}(v)$.

Next, suppose that $w \geq v$, and condition (C1) of Theorem 4 from the main paper is satisfied. If $w=v$, then $\operatorname{Depth}(y) \geq \operatorname{Depth}(w)=\operatorname{Depth}(v)$ gives a contradiction to (C1). If instead $w>v$, then $\operatorname{Depth}(w) \leq \operatorname{Depth}(y)<$ $\operatorname{Depth}(v)$, by $(\mathrm{C} 1)$. Suppose $w \in \operatorname{sib}(v)$, and consider $A_{w}$. Since $v \in S_{<}(w)$, there is a trek $\psi^{\prime}$ of the form $z \cdots v \leftrightarrow w$ with source $z \in Z_{w}$. But then $\operatorname{Depth}(z) \geq \operatorname{Depth}(v)>\operatorname{Depth}(w)$, which contradicts $(\mathrm{C} 1)$. Hence, we cannot have $w=v$ or $w \in S_{>}(v)$ if condition (C1) is true.

Next, suppose that $w=v$, and condition (C2) of Theorem 4 from the main paper is satisfied. If $\pi$ is a half-trek, then $v=w=y \in A_{v}$, a contradiction. If $w=v$ and $\pi$ is not a half-trek, then $y$ is a proper descendent of $w=v$, and so $y \in \operatorname{htr}(v)$. But then $(\mathrm{C} 2)$ requires that $\pi$ be a half-trek, a contradiction. Therefore, $w \neq v$ if condition (C2) is true.

Finally, suppose that $w>v$, and condition (C2) of Theorem 4 from the main paper is satisfied. If $w \in \operatorname{sib}(v)$, then $y=w$ or $y$ is a proper descendent of $w$. In either case, $y \in \operatorname{htr}(v) \cup S_{>}(v)$, and so $\pi$ must be a half-trek. It follows that $\pi$ has source node $w=y$, which implies that $w \prec v$ in the ordering specified by condition (C2). We now consider $A_{w}$. Since $v \in S_{<}(w)$, there is a trek $\psi^{\prime}$ of the form $z \cdots v \leftrightarrow w$ with source $z \in Z_{w}$. Then $v \in \operatorname{htr}(w)$ because of the half-trek $\pi^{\prime}$ from $w$ to $v$. Moreover, either $z=v$ or $z$ is a proper descendent of $v$. Therefore, $z \in \operatorname{htr}(w)$, and so $\psi^{\prime}$ must be a half-trek, implying that $z=v$. It follows that $v \in A_{w} \cap \operatorname{htr}(w)$, and so $v \prec w$ in the 
ordering specified by condition (C2). This is a contradiction. Therefore, we cannot have $w \in S_{>}(v)$ if condition (C2) is true.

We now prove the theorem.

THEOREM 5 (FROM MAIN PAPER). A GC-identifiable acyclic mixed graph is also HTC-identifiable.

Proof. First, consider the case that condition (C1) of Theorem 4 from the main paper. For each $v$, we can uniquely decompose each trek $\pi \in \Pi_{v}$ as

$$
y(\pi) \leftarrow \cdots \leftarrow y_{*}(\pi) \cdots t(\pi)
$$

where $y(\pi) \in Y_{v}$ is the source and $t(\pi) \in \operatorname{pa}(v)$ the target of $\pi$, and the subtrek $\pi_{*}$ from $y_{*}(\pi)$ to $t(\pi)$ is a half-trek. By Lemma $3, y_{*}(\pi) \neq v$ and $y_{*}(\pi) \notin \operatorname{sib}(v)$. Furthermore, for two distinct treks $\pi_{1}, \pi_{2} \in \Pi_{v}$, we must have $y_{*}\left(\pi_{1}\right) \neq y_{*}\left(\pi_{2}\right)$, because otherwise there would be a sided intersection between the extensions $\pi_{1}^{\prime}$ and $\pi_{2}^{\prime}$ of $\pi_{1}$ and $\pi_{2}$, respectively. Now define $Y_{v}^{*}=\left\{y_{*}(\pi): \pi \in \Pi_{v}\right\}$. Using the system of half-treks $\Phi_{v}=\left\{\pi_{*}: \pi \in \Pi_{v}\right\}$, we see that $Y_{v}^{*}$ satisfies the half-trek criterion with respect to $v$, for each $v$. Finally, define a total ordering $\prec$ on $V$ that agrees with the partial ordering induced by depth. Observe that for all $v, w$, it holds that $\operatorname{Depth}\left(y_{*}(\pi)\right) \leq$ $\operatorname{Depth}(y(\pi))<\operatorname{Depth}(v)$, by condition (C1). Hence, for any $y \in Y_{v}^{*} \cap \operatorname{htr}(v)$, we must have $\operatorname{Depth}(y)<\operatorname{Depth}(v)$, and so $y \prec v$. Consequently, the conditions of Theorem 1 from the main paper are satisfied, and the graph $G$ is HTC-identifiable.

Next, consider the case that condition (C2) of Theorem 4 from the main paper. For each $v$, by Lemma $3, Y_{v}$ is disjoint from $\operatorname{sib}(v) \cup\{v\}$. By the G-criterion, the system of treks $\Pi_{v}: Y_{v} \rightrightarrows \mathrm{pa}(v)$ has no sided intersection. By condition (C2), a trek $\pi \in \Pi_{v}$ is a half-trek whenever the source $y(\pi) \in$ $Y_{v} \cap \operatorname{htr}(v)$. Therefore, the set $Y_{v}$ satisfies the weak half-trek criterion with respect to $v$. Finally, take the ordering $\prec$ specified by condition (C2). For each $y \in Y_{v} \cap \operatorname{htr}(v)$, we must have $w \prec v$ for all $w \in Y_{v}$, by condition (C2). Therefore, using the weak half-trek method in Theorem 3 from the main paper, the graph $G$ is seen to be HTC-identifiable.

Proposition 3 (FROM MAIN PAPER). The acyclic mixed graph in Figure 2 from the main paper is not GC-identifiable.

Proof. First, note that the sibling sets $S_{<}(v)$ are unique because this graph $G$ has a unique topological ordering. Next, observe that with both $1 \rightarrow 2$ and $1 \leftrightarrow 2$ in the graph, $|\mathrm{pa}(2)|+\left|S_{<}(2)\right|=2$. But only node 1 
has depth smaller than node 2 . Therefore, $G$ cannot be GC-identifiable via condition (C1) of Theorem 4 from the main paper, and it remains to consider condition $(\mathrm{C} 2)$.

Node 2 We have $S_{<}(2)=\{1\}$ and must therefore find a set $Z_{2}=\{v\}$ such that there exists a trek $\pi$ of the form $v \leftarrow \cdots \leftarrow 1 \leftrightarrow 2$. If $v \in\{3,4,5\}$, then $v \in Z_{2} \cap \operatorname{htr}(2)$, and $\pi$ would need to be a half-trek, which is a contradiction. We conclude that $Z_{2}=\{1\}$, implying $1 \notin Y_{2}$. The parent set of node 2 is $\mathrm{pa}(2)=\{1\}$, and we must find $Y_{2}=\{v\}$ for a node $v \in\{3,4,5\}$ that is the source of a trek $\pi$ of the form $v \cdots 1 \rightarrow 2$. Since $\{3,4,5\} \subset \operatorname{htr}(2)$, the trek $\pi$ must be a half-trek. This restricts the choice to $v \in\{4,5\}$. Therefore, either $4 \in Y_{2} \cap \operatorname{htr}(2)$ or $5 \in Y_{2} \cap \operatorname{htr}(2)$. Hence, either $4 \prec 2$ or $5 \prec 2$.

Node 4 Starting from $3 \in S_{<}(4)$ and reasoning as for $Z_{2}$ before, we must have $3 \in Z_{4}$ and, consequently, $3 \notin Y_{4}$. Since pa(4) $=\{3\}$, we must have $Y_{4}=\{v\}$ with a trek $\pi$ of the form $v \cdots 3 \rightarrow 4$. The set $Z_{4}$ must contain a node $w$ at the source of a trek $w \cdots 1 \leftrightarrow 4$. Hence, $v=1$ cannot be in $Y_{4}$ since a sided intersection in the system of treks would be created. Therefore, we must have $v \in\{2,5\} \subset \operatorname{htr}(4)$, and so. It follows that either $2 \prec 4$ or $5 \prec 4$.

Node 5 We have $4 \in S_{<}(5)$. By the same reasoning as for $Z_{2}$ and $Z_{4}$, it holds that $4 \in Z_{5}$. Since $4 \in \operatorname{htr}(5)$, this means that $4 \prec 5$.

We conclude that a total ordering $\prec$ as required for GC-identifiability would have to satisfy either $4 \prec 5 \prec 4$, or $2 \prec 4 \prec 2$, or $2 \prec 4 \prec 5 \prec 2$. Consequently, no such ordering exists.

\section{Proofs for graph decomposition.}

Lemma 4. Let $v$ be a node in the mixed component $G^{\prime}$ of an acyclic mixed graph $G$. Consider the set $\operatorname{htr}(v)$ in $G$, and let $\operatorname{htr}^{\prime}(v)$ be the analogue in $G^{\prime}$. If there is a set $Y$ that satisfies the half-trek criterion with respect to $v$ in $G$, then there is a set $Y^{\prime}$ that satisfies the half-trek criterion with respect to $v$ in $G^{\prime}$, with $Y^{\prime} \cap \operatorname{htr}^{\prime}(v) \subset Y \cap \operatorname{htr}(v)$.

Proof. Let $V^{\prime}$ be the vertex set of $G^{\prime}$, and let $C^{\prime} \subset V^{\prime}$ be the vertex set of the bidirected connected component of $G$ that defined $G^{\prime}$. We may assume that $v \in C^{\prime}$, for otherwise $v$ has no parents in $G^{\prime}$ and the claims concern empty sets. Choose a system of half-treks $\Pi: Y \rightrightarrows \mathrm{pa}(v)$ with no sided intersection and with $Y \cap(\{v\} \cup \operatorname{sib}(v))=\emptyset$. Since pa $(v) \subset V^{\prime}$, each half-trek $\pi \in \Pi$ eventually visits only nodes in $V^{\prime}$. Now take $\Pi^{\prime}$ to be the set of half-treks obtained by retaining the longest subtrek of each half-trek 
$\pi \in \Pi$ that remains entirely in $G^{\prime}$ and contains the target of $\pi$. If $\pi^{\prime} \in \Pi^{\prime}$ is derived from $\pi \in \Pi$, then either (i) $\pi^{\prime}=\pi$ or (ii) $\pi^{\prime}$ is a directed path and its source $y^{\prime}$ is an element of $V^{\prime} \backslash C^{\prime}$.

First, we claim that $\Pi^{\prime}$ is a system of half-treks. In other words, we claim that the sources $y_{1}^{\prime}$ and $y_{2}^{\prime}$ of two distinct half-treks $\pi_{1}^{\prime}, \pi_{2}^{\prime} \in \Pi^{\prime}$ satisfy $y_{1}^{\prime} \neq$ $y_{2}^{\prime}$. Let $\pi_{1}$ and $\pi_{2}$ be the half-treks in $\Pi$ that yielded $\pi_{1}^{\prime}$ and $\pi_{2}^{\prime}$, respectively. Since $\Pi$ is without sided intersection, $y_{1}^{\prime} \neq y_{2}^{\prime}$ if both $\pi_{1}^{\prime}=\pi_{1}$ and $\pi_{2}^{\prime}=\pi_{2}$ (because then $y_{1}^{\prime}=y_{1}$ and $y_{2}^{\prime}=y_{2}$ ). If, without loss of generality, $\pi_{1}^{\prime} \neq \pi_{1}$, then $y_{1}^{\prime} \in \operatorname{Right}\left(\pi_{1}\right)$, and $y_{1}^{\prime} \notin C^{\prime}$. Now suppose $y_{1}^{\prime}=y_{2}^{\prime}$. Since $\Pi$ has no sided intersection, we must have $y_{2}^{\prime} \notin \operatorname{Right}\left(\pi_{2}\right)$. This implies that $\pi_{2}^{\prime}$ starts with a bidirected edge and, thus, $\pi_{2}^{\prime}=\pi_{2}$, and therefore $y_{2}^{\prime} \in C^{\prime}$, while $y_{1}^{\prime} \notin C^{\prime}$. Consequently, $y_{1}^{\prime} \neq y_{2}^{\prime}$.

Second, we claim that $\Pi^{\prime}$ has no sided intersections. Consider any $\pi_{1}, \pi_{2} \in$ $\Pi$. Since $\pi_{1}^{\prime}$ and $\pi_{2}^{\prime}$ are half-treks, Left $\left(\pi_{1}^{\prime}\right)=\left\{y_{1}^{\prime}\right\}$ and Left $\left(\pi_{2}^{\prime}\right)=\left\{y_{2}^{\prime}\right\}$. Above, we showed that $y_{1}^{\prime} \neq y_{2}^{\prime}$, and therefore $\operatorname{Left}\left(\pi_{1}^{\prime}\right) \cap \operatorname{Left}\left(\pi_{2}^{\prime}\right)=\emptyset$. Next we consider the right-hand sides. By definition of $\pi_{1}^{\prime}$ and $\pi_{2}^{\prime}$, we have $\operatorname{Right}\left(\pi_{1}^{\prime}\right) \subseteq \operatorname{Right}\left(\pi_{1}\right)$ and $\operatorname{Right}\left(\pi_{2}^{\prime}\right) \subseteq \operatorname{Right}\left(\pi_{2}\right)$. Therefore,

$$
\operatorname{Right}\left(\pi_{1}^{\prime}\right) \cap \operatorname{Right}\left(\pi_{2}^{\prime}\right) \subseteq \operatorname{Right}\left(\pi_{1}\right) \cap \operatorname{Right}\left(\pi_{2}\right)=\emptyset .
$$

Third, we claim that $\Pi^{\prime}$ satisfies the half-trek criterion with respect to $v$ in the component $G^{\prime}$. For this it remains to show that no source in $Y^{\prime}$ is equal to $v$ or a sibling of $v$. Indeed if the source $y^{\prime}$ of a half-trek $\pi^{\prime} \in$ $\Pi^{\prime}$ is in $\operatorname{sib}(v) \cup\{v\} \subset C^{\prime}$, then $\pi^{\prime}=\pi$ and we have a contradiction to $Y \cap(\{v\} \cup \operatorname{sib}(v))=\emptyset$.

Finally, we claim that $Y^{\prime} \cap \operatorname{htr}^{\prime}(v) \subset Y \cap \operatorname{htr}(v)$. Since $G^{\prime}$ is a subgraph of $G$, we have $\operatorname{htr}^{\prime}(v) \subset \operatorname{htr}(v)$. Our claim thus holds because all nodes in $Y^{\prime} \backslash Y$ are in $V^{\prime} \backslash C^{\prime}$ and there are no directed edges in $G^{\prime}$ that point to nodes in $V^{\prime} \backslash C^{\prime}$.

Lemma 5. Let $G_{1}, \ldots, G_{k}$ be the mixed components of an acyclic mixed graph $G$. Suppose that $G_{1}, \ldots, G_{k}$ are not HTC-infinite-to-one. Then $G$ is not HTC-infinite-to-one.

Proof. For each $j \in[k]$, choose a family $\left(Y_{v}^{(j)}: v \in V_{j}\right)$ where each set $Y_{v}^{(j)} \subset V_{j}$ satisfies the half-trek criterion with respect to $v$ in the component $G_{j}$, and for all $v, w \in V_{j}$, either $v \notin Y_{w}^{(j)}$ or $w \notin Y_{v}^{(j)}$. Such a family exists by the assumption.

For $v \in V$, let $j(v)$ be the unique index in $[k]$ with $v \in C_{j(v)}$. Define $Y_{v}=Y_{v}^{(j(v))}$. The original graph $G$ is seen not to be HTC-infinite-to-one, if the following two claims are proven: 
(a) in $G$, each set $Y_{v}$ satisfies the half-trek criterion with respect to its indexing node $v$;

(b) for each $v \neq w \in V$, either $v \notin Y_{w}$ or $w \notin Y_{v}$.

Proof of claim (a): Fix any $v$ and abbreviate $j=j(v)$. By definition, $Y_{v}=Y_{v}^{(j(v))}$ satisfies the half-trek criterion with respect to $v$ in $G_{j}$. This implies that there is a system $\Pi$ of half-treks with no sided intersection from $Y_{v}$ to $\mathrm{pa}(v) \cap V_{j}$, and that $Y_{v} \subset V_{j} \backslash\left(v \cup\left(\operatorname{sib}(v) \cap V_{j}\right)\right)$. However, by definition of $G_{j}$, we know $\operatorname{pa}(v) \subseteq V_{j}$ and $\operatorname{sib}(v) \subseteq V_{j}$. Hence, $Y_{v}$ satisfies the half-trek criterion with respect to $v$, in $G$.

Proof of claim (b): Fix any two nodes $v \neq w$. If $j(v)=j(w)$, then by assumption, either $v \notin Y_{w}^{(j(w))}=Y_{w}$ or $w \notin Y_{v}^{(j(v)}=Y_{v}$. Now suppose $j(v) \neq j(w)$, and $w \in Y_{v}$. Then $w \in V_{j(v)} \backslash C_{j(v)}$, implying that there exists a directed path from $w$ to $v$ in $G_{j(v)}$. Similarly, if $v \in Y_{w}$, then there is a directed path from $v$ to $w$ in $G_{j(w)}$. Since the directed part of $G$ is acyclic, this is a contradiction.

Lemma 6. Suppose $G$ is not HTC-infinite-to-one. Then $G_{j}$ is not HTCinfinite-to-one for all $j \in[k]$.

Proof. If $G$ is not HTC-infinite-to-one, then there exists a family $\left(Y_{v}: v \in V\right)$ of subsets of $V$, such that for each $v, Y_{v}$ satisfies the half-trek criterion with respect to $v$, and for all $v \neq w$, either $v \notin Y_{w}$ or $w \notin Y_{v}$.

Now fix any $j \in[k]$. For each $v \in V_{j}$, we adopt the construction from the proof of Lemma 4 to obtain a system of half-treks $\Pi_{v}^{\prime}: Y_{v}^{\prime} \rightrightarrows \operatorname{pa}(v)$ in $G_{j}$ that shows that $Y_{v}^{\prime}$ satisfies the half-trek criterion with respect to $v$ in $G_{j}$. For each $v \in V_{j} \backslash C_{j}, v$ has no parents in $G^{\prime}$, and so we can define $Y_{v}^{\prime}=\emptyset$.

Now consider any $v, w \in V_{j}$. Suppose for a contradiction that $v \in Y_{w}^{\prime}$ and $w \in Y_{v}^{\prime}$. This implies $Y_{v}^{\prime}, Y_{w}^{\prime} \neq \emptyset$, and so $v, w \in C_{j}$. In this case, $v$ is the source of some half-trek in $\Pi_{w}^{\prime}$. But then $v \in C_{j}$ implies that this half-trek was unchanged when constructing $\Pi_{w}^{\prime}$, and thus $v$ is also in $Y_{w}$. The same argument shows that $w \in Y_{v}$, which contradicts the assumption made for our claim.

6. Proofs for algorithms. We first state some known properties of the max-flow problem for a directed graph $G=(V, D)$ with source node $s$, sink node $t$, and node and edge capacity functions $c_{V}$ and $c_{D}$, respectively. Our references are [5] and [1]. Recall that we called a pair edges reciprocal if it is consists the two edges $v \rightarrow w$ and $w \rightarrow v$ for distinct nodes $v \neq w$. If all node capacities are infinite, that is, $c_{V}(v)=\infty$ for all $v \in V$, then we say that there are no node capacity bounds. 
(a) For any graph $G$, the max-flow problem on $\left(G, s, t, c_{V}, c_{D}\right)$ can be converted into a max-flow problem on a digraph $G^{\prime}=\left(V^{\prime}, D^{\prime}\right)$ with no node capacity bounds and no reciprocal edges, such that $\left|V^{\prime}\right| \leq 2|V|+r$ and $\left|D^{\prime}\right| \leq|V|+\frac{3}{2}|D|$, where $r \leq|D| / 2$ is the number of reciprocal edge pairs in $G$.

(b) If $G$ has no reciprocal edges and there are no node capacity bounds, then the computational complexity of any max-flow problem for $G$ is $\mathcal{O}\left(|V|^{3}\right)$.

(c) If the capacity functions $c_{V}$ and $c_{D}$ are both integer-valued, with $c_{V}(v)=\infty$ allowed, then the maximum flow size on $\left(G, s, t, c_{V}, c_{D}\right)$ can be achieved with an integer-valued flow $f$ (with no increase in computational complexity).

Combining these facts, we see that the computational complexity of solving a max-flow problem on a network $G=(V, D)$ with $r$ reciprocal edge pairs and both node and edge capacities is at most $\mathcal{O}\left((|V|+r)^{3}\right)$.

Next we prove the theorems that justify Algorithm 1 from the main paper, which decides HTC-identifiability.

TheOrEm 6 (From MAIN PAPER). Given a mixed graph $G=(V, D, B)$, a node $v \in V$, and a subset of "allowed" nodes $A \subseteq V \backslash(\{v\} \cup \operatorname{sib}(v))$, there exists a set $Y \subseteq A$ satisfying the half-trek criterion with respect to $v$ if and only if the flow network $G_{\text {flow }}(v, A)$ has maximum flow equal to $|\mathrm{pa}(v)|$.

Proof. If $Y \subseteq A$ satisfies the half-trek criterion with respect to $v$, then we have a system of $|\mathrm{pa}(v)|$ half-treks with no sided intersection. We write the half-treks as

$$
\pi_{k}: y_{k} \circ \mathrm{o}_{k, 1} \rightarrow z_{k, 2} \rightarrow \ldots \rightarrow k, \quad k \in \mathrm{pa}(v),
$$

where the symbol $y_{k} \circ-\circ z_{k, 1}$ represents either $y_{k}=z_{k, 1}$ or $y_{k} \leftrightarrow z_{k, 1}$. The set $Y=\left\{y_{k}: k \in \mathrm{pa}(v)\right\}$ comprises the left-hand sides of the half-treks.

Now, for each $k$, add a flow of size 1 along the path

$$
\tilde{\pi}_{k}: s \rightarrow L\left(y_{k}\right) \rightarrow R\left(z_{k, 1}\right) \rightarrow R\left(z_{k, 2}\right) \rightarrow \ldots \rightarrow R(k) \rightarrow t
$$

in the network $G_{\text {flow }}(v, A)$. Clearly, the resulting total flow size is $|\mathrm{pa}(v)|$, and it remains to check that no capacity is exceeded. Recall that edges have infinite capacity, as do the nodes $s$ and $t$. For any $a \in A$, the node $L(a)$ appears in $\tilde{\pi}_{k}$ if and only if $a \in \operatorname{Left}\left(\pi_{k}\right)$, and similarly, for any $w \in V$, the node $R(w)$ appears in $\tilde{\pi}_{k}$ if and only if $w \in \operatorname{Right}\left(\pi_{k}\right)$. Since the system of half-treks $\left\{\pi_{k}\right\}$ has no sided intersection, it follows that no node is used more than once in the flow. Hence, capacity constraints are satisfied. 
Now suppose a flow $f$ of size $|\mathrm{pa}(v)|$ exists. By the properties of the maxflow problem with integer-valued capacities [5], this flow can be decomposed into $|\mathrm{pa}(v)|$ directed paths from $s$ to $t$ with flow size 1 along each path. We denote these paths as $\tilde{\pi}_{k}, k \in \mathrm{pa}(v)$. By definition of the node capacity function $c_{V}$, each node $L(a)$ and each node $R(w)$ can appear at most once in this system of paths. Now consider the path $\tilde{\pi}_{k}$. By the construction of $G_{\text {flow }}(v, A)$, it must be of the form

$$
\pi_{k}: s \rightarrow L\left(a_{k}\right) \rightarrow R\left(z_{k, 1}\right) \rightarrow R\left(z_{k, 2}\right) \rightarrow \ldots \rightarrow R\left(p_{k}\right) \rightarrow t
$$

with $a_{k} \in A, p_{k} \in \mathrm{pa}(v)$ and $z_{k, 1}, z_{k, 2}, \ldots$ some nodes in $V$. By definition of $G_{\text {flow }}(v, A)$, this defines the half-trek

$$
\pi_{k}: a_{k} \circ-\circ z_{k, 1} \rightarrow z_{k, 2} \rightarrow \ldots \rightarrow p_{k}
$$

in $G$, where $a_{k} \circ-\circ z_{k, 1}$ represents either $a_{k}=z_{k, 1}$ or $a_{k} \leftrightarrow z_{k, 1}$ as appropriate. Note that $\operatorname{Left}\left(\pi_{k}\right)=\left\{a_{k}\right\}$ and $\operatorname{Right}\left(\pi_{k}\right)=\left\{z_{k, 1}, z_{k, 2}, \ldots, p_{k}\right\}$. Therefore, since the nodes $L\left(a_{k}\right)$ and $R\left(z_{k, 1}, R\left(z_{k, 2}\right), \ldots, R\left(p_{k}\right)\right.$ have capacity 1 in $G_{\text {flow }}(v, A)$, the system of half-treks $\left\{\pi_{k}: k \in \mathrm{pa}(v)\right\}$ corresponding to the maximum flow cannot have any sided intersection. Hence, we have constructed a system of half-treks with no sided intersection from $Y \subseteq A$ to $\operatorname{pa}(v)$, where $Y=\left\{a_{k}: f\left(s, L\left(a_{k}\right)\right)=1\right\}$.

Theorem 7 (From main PAPER). A mixed graph $G=(V, D, B)$ is $H T C$-identifiable if and only if Algorithm 1 from the main paper returns "yes". Furthermore, the algorithm has complexity at most $\mathcal{O}\left(|V|^{2}(|V|+r)^{3}\right)$, where $r \leq|D| / 2$ is the number of reciprocal edge pairs in $D$.

Proof. First, we calculate the algorithm's complexity. We claim that the algorithm terminates after at most $|V|$ passes through the nodes. Indeed, in each pass either a node is added to the set SolvedNodes or the algorithm terminates. Therefore, either all nodes get added in at most $|V|$ passes, or the termination occurs no later than the $|V|$-th pass. Hence, we must find a maximum flow at most $|V|^{2}$ times, on a graph with at most $2|V|+2$ vertices, at most $3|V|+|D|+|B|$ edges, and the same number of reciprocal edge pairs as $G$. Each individual max-flow problem having complexity $\mathcal{O}\left((|V|+r)^{3}\right)$, the total complexity is $\mathcal{O}\left(|V|^{2}(|V|+r)^{3}\right)$.

Next, to show that the algorithm indeed determines HTC-identifiability, suppose that $G$ is HTC-identifiable. Then by definition, we have an ordering $\prec$ on $V$, and for each node $v$, a set $Y_{v}$ satisfying the half-trek criterion with respect to $v$, such that $w \in Y_{v} \cap h \operatorname{tr}(v)$ implies $w \prec v$. We now claim that after at most $k+1$ passes through the for-loop in the algorithm, any node $v$ with 
depth $k$ under the ordering $\prec$ has been added to SolvedNodes. Arguing by induction, suppose all nodes $w$ with $\operatorname{depth}(w)<k$ under the ordering $\prec$ are already in SolvedNodes, and we are now testing node $v$ with $\operatorname{depth}(v)=k$. Take any $w \in Y_{v}$. By definition, $w \notin\{v\} \cup \operatorname{sib}(v)$. If $w \in \operatorname{htr}(v)$ then $w \prec v$ and so $\operatorname{depth}(w)<k$, and thus $w \in$ SolvedNodes $\subseteq A$. If instead $w \notin \operatorname{htr}(v)$ then $w \in A$ by definition. Therefore, $Y_{v} \subseteq A$, and so by the maximum flow result from Theorem 6 from the main paper, we will add $v$ to SolvedNodes. By induction, we see that we will have SolvedNodes $=V$ after at most $m$ iterations.

Conversely, suppose the algorithm finds SolvedNodes $=V$. Define an ordering $\prec$ on $V$ by ordering the nodes in the order that they were added to SolvedNodes. It remains to obtain the family $\left(Y_{v}: v \in V\right)$. Fix a node $v \in V$. When $v$ was added in the algorithm, the maximum flow size in $G_{\text {flow }}(v, A)$ must have been equal to $|\mathrm{pa}(v)|$. By Theorem 6 from the main paper, this means that there is a set $Y_{v} \subseteq A$ with $\left|Y_{v}\right|=|\mathrm{pa}(v)|$ that satisfies the halftrek criterion with respect to $v$. Furthermore, by definition of $A$ at this stage of the algorithm, for any $w \in A$, we have $w \notin\{v\} \cup \operatorname{sib}(v)$. In addition, we either have $w \in$ SolvedNodes already (i.e., $w \prec v$ ) or $w \notin \operatorname{htr}(v)$. Applying this reasoning to all $v$, we see that $G$ is HTC-identifiable.

Finally, we prove that the problem of determining whether $G$ is HTCinfinite-to-one, can be reformulated as a max-flow problem on the graph $G_{\text {flow }}$ defined in Section 6 from the main paper.

TheOREM 8 (From MAIN PAPER). A mixed graph $G=(V, D, B)$ is $H T C$-nonidentifiable if and only if $G_{\text {flow }}$ has maximum flow size less than $|D|=\sum_{v \in V}|\mathrm{pa}(v)|$. The computational complexity of solving this max-flow problem is $\mathcal{O}\left(|V|^{3}(|V|+r)^{3}\right)$, where $r \leq|D| / 2$ is the number of reciprocal edge pairs in $G$.

Proof. Since $G_{\text {flow }}$ has at most $\frac{3}{2}|V|^{2}+2$ nodes and $|V| \cdot r$ reciprocal edge pairs, the complexity bound follows immediately. It remains to show that the max-flow problem in $G_{\text {flow }}$ gives us the HTC-infinite-to-one status of $G$.

First, suppose $G$ is not HTC-infinite-to-one. Then there exists a family of sets $\left(Y_{v}: v \in V\right)$ such that $Y_{v}$ satisfies the half-trek criterion with respect to $v$ for each $v$, and $w \in Y_{v}$ implies $v \notin Y_{w}$. Consequently, for each $v$, there is a system of $|\mathrm{pa}(v)|$ half-treks with no sided intersection. We write the half-treks as

$$
\pi_{(v, k)}: y_{(v, k)} \circ \mathrm{O}_{(v, k), 1} \rightarrow z_{(v, k), 2} \rightarrow \ldots \rightarrow k, \quad k \in \mathrm{pa}(v)
$$


where $y_{(v, k)} \in Y_{v}$ and $y_{(v, k)} \circ-\circ z_{(v, k), 1}$ means either $y_{(v, k)}=z_{(v, k), 1}$ or $y_{(v, k)} \leftrightarrow z_{(v, k), 1}$. To construct a flow in $G_{\text {flow }}$, add flow of size 1 to along the path

$$
\tilde{\pi}_{(v, k)}: s \rightarrow L\left\{v, y_{(v, k)}\right\} \rightarrow R_{v}\left(z_{(v, k), 1}\right) \rightarrow R_{v}\left(z_{(v, k), 2}\right) \rightarrow \ldots \rightarrow R_{v}(k) \rightarrow t,
$$

for each pair of node and parent $(v, k)$. Clearly, this results in total flow of size $\sum_{v}|\mathrm{pa}(v)|=|D|$. To check the capacity constraints, we make the observations:

(a) Fix any pair of nodes $v$ and $w$ with $v \nLeftarrow<w$ in $G$. If path $\tilde{\pi}_{(v, k)}$ passes through $L\{v, w\}$, then $w \in Y_{v}$. It follows that $v \notin Y_{w}$, which implies that $L\{v, w\}$ does not appear in any path $\tilde{\pi}_{(w, l)}$. For a node $u$ different from both $v$ and $w$, we cannot have $L\{v, w\} \in \tilde{\pi}_{(u, l)}$, by definition. Finally, for $l \neq k$, the node $L\{v, w\}$ cannot appear in $\tilde{\pi}_{(v, l)}$ because nodes in $Y_{v}$ cannot be used multiple times in the system of half-treks $\pi_{(v, k)}, k \in \mathrm{pa}(v)$.

(b) Fix any two nodes $v, w$ in $G$. The node $R_{v}(w)$ can appear in $\tilde{\pi}_{(u, k)}$ only if $u=v$. Furthermore, $R_{v}(w)$ node cannot appear in $\tilde{\pi}_{(v, k)}$ and $\tilde{\pi}_{(v, l)}$ for $l \neq k$, because this would create a right-hand-sided intersection between $\pi_{(v, k)}$ and $\pi_{(v, l)}$.

We conclude that the capacity constraints on both the left-hand nodes $L\{v, w\}$ and the right-hand nodes $R_{v}(w)$ are satisfied.

Conversely, suppose that $G_{\text {flow }}$ has a flow of size $|D|=\sum_{v}|\mathrm{pa}(v)|$. Since $|D|$ is equal to the number of edges incoming to the sink $t$ in $G_{\text {flow }}$, we must have one path for each such edge, and so we have a maximum flow that is the sum of flows along directed paths $\tilde{\pi}_{(v, k)}$, indexed by all pairs $(v, k)$ with $v \in V$ and $k \in \mathrm{pa}(v)$. By definition of $G_{\text {flow }}$, these paths must be of the form

$$
\tilde{\pi}_{(v, k)}: s \rightarrow L\left\{v, y_{(v, k)}\right\} \rightarrow R_{v}\left(z_{(v, k), 1}\right) \rightarrow R_{v}\left(z_{(v, k), 2}\right) \rightarrow \ldots \rightarrow R_{v}(k) \rightarrow t,
$$

where $y_{(v, k)}$ is not a sibling of $v$. Hence, $\tilde{\pi}_{(v, k)}$ corresponds to a half-trek in $G$, namely,

$$
\pi_{(v, k)}: y_{(v, k)} \circ \mathrm{O}_{(v, k), 1} \rightarrow z_{(v, k), 2} \rightarrow \ldots \rightarrow k .
$$

Defining $Y_{v}=\left\{y_{(v, k)}: k \in \mathrm{pa}(v)\right\}$ for each $v$, we follow the same reasoning as above, and see that $Y_{v}$ satisfies the half-trek criterion with respect to $v$ for each $v$, and that $w \in Y_{v}$ implies $v \notin Y_{w}$. Therefore, $G$ is not HTC-infiniteto-one. 


\section{Proofs for the weak half-trek criterion.}

LEMMA 1 (FROM MAIN PAPER). Suppose the set $W \subset V$ satisfies the weak half-trek criterion with respect to some node $v$. Then there exists a set $Y$ satisfying the half-trek criterion with respect to $v$, such that $Y \cap \operatorname{htr}(v)=$ $W \cap \operatorname{htr}(v)$.

Proof. Let $\Pi: W \rightrightarrows \mathrm{pa}(v)$ be a system of treks satisfying the conditions of the weak half-trek criterion. Let $r$ be the number of treks in $\Pi$ which are not half-treks, and suppose $r>0$. Using induction, it suffices to show that there is a set $W^{\prime}$ satisfying the weak half-trek criterion with respect to $v$ via some trek system $\Pi^{\prime}$ with no more than $r-1$ treks that are not half-treks, and for which $W^{\prime} \cap \operatorname{htr}(v)=W \cap h \operatorname{tr}(v)$.

Take any $w \in W$ for which the trek $\pi \in \Pi$ with source $w$ is not a half-trek. By the definition of the weak half-trek criterion, this implies that $w \notin \operatorname{htr}(v)$. Let $w^{\prime} \neq w$ be the (unique) node in the left-hand side of $\pi$ that is closest to the target of $\pi$, which we denote $t(\pi)$. The trek $\pi$ has the structure

$$
w \leftarrow \cdots \leftarrow w^{\prime} \cdots t(\pi) .
$$

Let $\pi^{\prime}$ be the subtrek from $w^{\prime}$ to $t(\pi)$. Then $\pi^{\prime}$ is a half-trek. Since $w$ is a descendent of $w^{\prime}$ and $w \notin h \operatorname{tr}(v)$, this implies $w^{\prime} \notin \operatorname{htr}(v)$ and $w \notin \operatorname{sib}(v)$. Moreover, $w^{\prime} \notin W \backslash\{w\}$ because $\pi$ has no sided intersection and $w^{\prime}$ is in the left-hand side of $\pi$.

Define $W^{\prime}=(W \backslash\{w\}) \cup\left\{w^{\prime}\right\}$ and $\Pi^{\prime}=(\Pi \backslash\{\pi\}) \cup\left\{\pi^{\prime}\right\}$. Since the original system of treks $\Pi$ had no sided intersection, the new system of treks $\Pi^{\prime}$ also has no sided intersection. Precisely $r-1$ of the treks in $\Pi^{\prime}$ are not half-treks. Furthermore, since $w, w^{\prime} \notin \operatorname{htr}(v)$, we have $W^{\prime} \cap \operatorname{htr}(v)=W \cap \operatorname{htr}(v)$, as needed to be shown.

TheOREM 3 (FROM MAIN PAPER) (Weak HTC). Theorems 1 and 2 from the main paper hold when using the weak half-trek criterion instead of the half-trek criterion. Moreover, a graph $G$ can be proved to be rationally identifiable (or generically infinite-to-one) using the weak half-trek criterion if and only if $G$ is HTC-identifiable (or HTC-infinite-to-one).

Proof. It is sufficient to show the following two facts:

(a) If $G$ can be proved to be rationally identifiable using the weak half-trek criterion, then $G$ is HTC-identifiable.

(b) If $G$ cannot be proved to be generically infinite-to-one using the weak half-trek criterion, then $G$ is not HTC-infinite-to-one. 
Part (a). A graph $G$ can be proved to be rationally identifiable using the weak half-trek criterion if there is, for each $v$, a set of nodes $W_{v}$ satisfying the weak half-trek criterion with respect to $v$, and an ordering $\prec$ such that $w \prec v$ for any $w \in W_{v} \cap \operatorname{htr}(v)$. By Lemma 1 from the main paper (proved above), for each $v$, there is then also a set $Y_{v}$ satisfying the half-trek criterion with respect to $v$, with $Y_{v} \cap \operatorname{htr}(v)=W_{v} \cap \operatorname{htr}(v)$. Therefore, $G$ is seen to be HTC-identifiable using the same ordering $\prec$.

Part (b). If $G$ cannot be proved to be generically infinite-to-one using the weak half-trek criterion, then there is a family $\left(W_{v}: v \in V\right)$, such that each $W_{v}$ satisfies the weak half-trek criterion with respect to $v$, and $v \in W_{w}$ implies $w \notin W_{v}$. Using Lemma 1 from the main paper, we can find, for each $v$, a set $Y_{v}$ that satisfies the half-trek criterion with respect to $v$ and for which $Y_{v} \cap \operatorname{htr}(v)=W_{v} \cap \operatorname{htr}(v)$. Now suppose $v \in Y_{w}$ for two nodes $v, w \in V$. This means that $v \notin S(w) \cup\{w\}$ and there is a half-trek $\pi$ with source $v$ and target $w$, which implies that $w \in \operatorname{htr}(v)$. If also $w \in Y_{v}$, then $w \in Y_{v} \cap \operatorname{htr}(v)=W_{v} \cap \operatorname{htr}(v)$. By symmetry, we also get $v \in W_{w}$. This contradicts our assumption, and so $w \notin Y_{v}$. This proves that $G$ cannot be proved to be generically infinite-to-one using the half-trek criterion.

8. Algebraic techniques for proving and disproving identifibability. In the proofs of our half-trek criteria we have made extensive use of the equations (9.2) from the main paper, which we restate here:

$$
\left[(I-\Lambda)^{T} \Sigma(I-\Lambda)\right]_{v w}=0 \quad \forall(v, w) \notin B \text { and } v \neq w .
$$

In this final section we discuss the algebro-geometric and computational content of these equations. In what follows, we write $\lambda$ and $\omega$ for tuples of variables representing the non-zero entries of $\Lambda$ and $\Omega$, and $\sigma$ for a tuple of variables representing the entries of $\Sigma$. We will also need the rational function $\delta:=\operatorname{det}(I-\Lambda)^{-1}$; in the acyclic case, $\delta$ is just the constant 1 . The entries of the inverse $(I-\Lambda)^{-1}$ lie in the ring $\mathbb{R}[\lambda, \delta]$ of polynomials in $\lambda$ and $\delta$. The first observation is the following.

Proposition 1. The left-hand sides of the equations (8.1) generate a radical ideal in the ring $\mathbb{R}[\lambda, \sigma, \delta]$.

We apply the theory of elimination ideals; see Chapter 3 of [2].

Proof. Recall the parametrization equation (1.4) from the main paper, namely,

$$
\Sigma-(I-\Lambda)^{-T} \Omega(I-\Lambda)^{-1}=0,
$$


and interpret the individual entries of the matrix on the left-hand side as generators of an ideal $\mathcal{J}$ in $\mathbb{R}[\lambda, \sigma, \omega, \delta]$. The ideal $\mathcal{J}$ is radical because it is the ideal of the graph of the parametrization $\phi_{G}$. Let $\mathcal{I}$ be the ideal generated by the left-hand sides of (8.1). We claim that $\mathcal{I}=\mathcal{J} \cap \mathbb{R}[\lambda, \sigma, \delta]$; the fact that $\mathcal{J}$ is radical then implies that $\mathcal{I}$ is radical as well.

The containment $\mathcal{I} \subseteq \mathcal{J} \cap \mathbb{R}[\lambda, \sigma, \delta]$ is immediate because $\mathcal{J}$ contains the entries of the matrix obtained by multiplying (8.2) from the left by $(I-\Lambda)^{T}$ and from the right by $(I-\Lambda)$, and among these entries are the generators of $\mathcal{I}$.

For the converse, let $f \in \mathcal{J} \cap \mathbb{R}[\lambda, \sigma, \delta]$. Let $\tilde{\Omega}$ be a symmetric matrix full of auxiliary new variables $\tilde{\omega}_{i j}=\tilde{\omega}_{j i}$, even at positions that do not correspond to bidirected edges, and let $g \in \mathbb{R}[\lambda, \tilde{\omega}, \delta]$ be the polynomial obtained from $f$ by substituting the entries of

$$
(I-\Lambda)^{-T} \tilde{\Omega}(I-\Lambda)^{-1}
$$

for $\sigma$. Since $f$ lies in $\mathcal{J}$, the polynomial $g$ becomes zero when setting all variables $\tilde{\omega}_{u v}$ with $u \neq v,(u, v) \notin B$ equal to zero. This means that $g$ lies in the ideal generated by these variables, i.e.,

$$
g=\sum_{u \neq v,(u, v) \notin B} h_{i} \cdot \tilde{\omega}_{u v}
$$

for suitable coefficients $h_{i} \in \mathbb{R}[\lambda, \tilde{\omega}, \delta]$. Re-substituting $(I-\Lambda)^{T} \Sigma(I-\Lambda)$ for $\tilde{\Omega}$ on the left-hand side yields $f$, and performing the same substitution on the right yields an $\mathbb{R}[\lambda, \sigma, \delta]$-linear combination of the generators of $\mathcal{I}$. It follows that $\mathcal{I}=\mathcal{J} \cap \mathbb{R}[\lambda, \sigma, \delta]$.

We continue to write $\mathcal{I}$ and $\mathcal{J}$ for the two ideals featuring in the proof just given. In more geometric language, the proposition and its proof show that $\mathcal{I}$ is the ideal of all polynomials vanishing identically on the projection of the graph of $\phi_{G}$ into the principal open subset of $(\lambda, \sigma)$-space where $\delta$ is defined.

LEMMA 7. The parameter $\lambda_{u v}$ is rationally identifiable if and only if $\mathcal{I}$ contains an element of the form $a(\sigma) \lambda_{u v}-b(\sigma)$ with $a, b \in \mathbb{R}[\sigma]$ and a not identically zero on the linear structural equation model given by the graph $G$.

In fact, in this case $b$ will not be identically zero on the model, either.

Proof. By definition, if $\lambda_{u v}$ is rationally identifiable, then there is a rational function $b(\sigma) / a(\sigma) \in \mathbb{R}(\sigma)$ which upon substituting for $\sigma$ the entries 
of $(I-\Lambda)^{-T} \Omega(I-\Lambda)$ becomes equal to $\lambda_{u v}$; in particular, this substitution must be well-defined, so that $a(\sigma)$ does not vanish identically on the model. This means that the polynomial $a(\sigma) \lambda_{u v}-b(\sigma)$ lies in the ideal $\mathcal{J}$ of the graph of the parametrization. Since it only depends on $\lambda$ and $\sigma$, it lies in $\mathcal{I}$ (see the proof of Proposition 1). Conversely, if $a, b$ are as in the lemma, then $b / a$ is a rational function identifying $\lambda_{u v}$ from $\sigma$.

Lemma 7 yields the following algorithm for checking rational identifiability of a graph $G$.

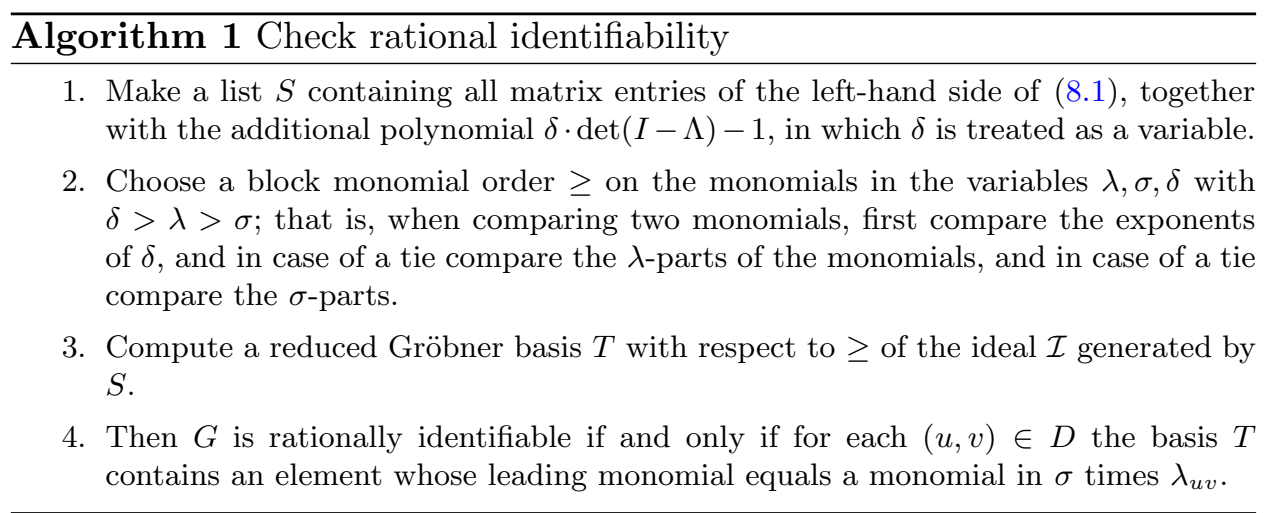

We now prove the correctness of the algorithm.

Proof. If $T$ contains a polynomial $f_{u v}$ whose leading monomial equals $\lambda_{u v}$ times a monomial in $\sigma$, then $f_{u v}$ is of the form $a(\sigma) \lambda_{u v}-b(\sigma, \lambda)$, where $b$ only contains $\lambda$-variables smaller than $\lambda_{u v}$. Moreover, $a$ does not vanish identically on the model (or else $a$ would be in $\mathcal{I}$ and hence $f_{u v}$ would not be reduced). Therefore, $\lambda_{u v}$ can be rationally identified if all smaller $\lambda$-variables can. Hence, if we assume that $T$ contains such a polynomial for all $(u, v) \in D$, then $G$ is rationally identifiable.

Conversely, if $\lambda_{u v}$ is rationally identified by $b(\sigma) / a(\sigma)$, then $a(\sigma) \lambda_{u v}-$ $b(\sigma) \in \mathcal{I}$ by Lemma 7 . Replace $a$ by its reduction modulo $T$; this reduction is non-zero since $a$ does not vanish identically on the model, and it contains only the variables $\sigma$ because of the choice of monomial order. Now the leading monomial of $a(\sigma) \lambda_{u v}-b(\sigma)$ equals $\lambda_{u v}$ times the leading monomial of $a$, and it is divisible by the leading monomial of some element $f$ of $T$. Then $f$ has leading monomial $\lambda_{u v}$ times some monomial in $\sigma$, as required.

The reduced Gröbner basis $T$ contains more information than is used in Step (4) of the algorithm just described. Indeed, straightforward mod- 
ifications of Step (4) can be used to test whether the parametrization is generically finite-to-one, and to find the degree of identifiability $I D(G)$.

Algorithm 1 is only a minor modification of the algorithm for rational identifiability in [6], which uses the equations in (8.2) rather than those in (8.1). In the acyclic case, the equations in (8.2) involve expanding the $\sigma_{v w}$ as polynomials in terms of the $\lambda$ - and $\omega$-variables using the Trek Rule (2.3) from the main paper. It is easy to construct families of acyclic graphs where the bit-size of those expanded polynomials grows exponentially with the size of the graph, so that in [6] the input to Buchberger's algorithm is already not polynomial in the size of $G$. By contrast, the bit-size of the cubic equations in (8.1) is clearly of size polynomial in the size of $G$, while in the acyclic case the additional polynomial $\delta \cdot \operatorname{det}(I-\Lambda)-1$ reduces to $\delta-1$. This reduction in bit-size, degree, and number of variables seems a significant theoretical improvement upon [6]. On the other hand, the worstcase size of a Gröbner basis is known to be doubly exponential in the size of the input $[4,7]$, and although we have not tried to construct a family of graphs for which the equations in (8.1) attain that worst-case size, in practice our algorithm does not seem to tackle graphs of sizes much beyond those studied in [6]. Moreover, in the cyclic case our algorithm does require symbolically expanding $\operatorname{det}(I-\Lambda)$, whose bit-size is again not polynomially bounded in the size of $G$.

For large-scale computations such as those in Section 7 from the main paper, we therefore used a randomized version in which the variables $\sigma$ are replaced by the numerical values of the entries of randomly chosen matrices in the model. In other words, for random choices of $\Lambda_{0} \in \mathbb{R}_{\mathrm{reg}}^{D}$ and $\Omega_{0} \in$ $P D(B)$, we compute a reduced Gröbner basis for the equation system

$$
\begin{aligned}
{\left[(I-\Lambda)^{T} \phi_{G}\left(\Lambda_{0}, \Omega_{0}\right)(I-\Lambda)\right]_{v w} } & =0 \quad \forall(v, w) \notin B \text { and } v \neq w \\
\delta \cdot \operatorname{det}(I-\Lambda)-1 & =0,
\end{aligned}
$$

under a block monomial order with $\delta>\lambda$. The reduced Gröbner basis informs us about the dimension and cardinality of the solution set of (8.3) and (8.4) over $\mathbb{C}_{\text {reg }}^{D} \times \mathbb{C}_{\mathrm{sym}}^{m \times m}$, and readily yields the degree of identifiability $I D(G)$. In particular, the basis corresponds to a linear equation system with unique solution if and only if the graph is rationally identifiable. Formally, the claims in the last sentences hold with probability one, if $\left(\Lambda_{0}, \Omega_{0}\right)$ is drawn from a continuous probability distribution. In practice, we generate random integer-valued matrices that are then processed in a computer algebra system such as Singular [3]. To guard against occasional false conclusions from random draws that yield matrices in special position, we repeat the randomized calculation several times for each graph. 


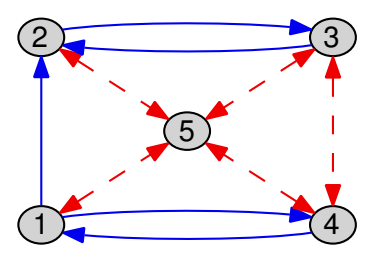

FIG 1. Cyclic graph exemplifying the need to remove points $(\Lambda, \Omega)$ with $\operatorname{det}(I-\Lambda)=0$ in algebraic computations.

Finally, we stress with our last example that the equation (8.4) cannot be omitted when studying cyclic graphs, even when $\Lambda_{0}$ is chosen to be in $\mathbb{R}_{\text {reg }}^{D}$.

EXAMPLE 1. For the graph $G$ in Figure 1, a run of Algorithm 1 without specializing values shows that the ideal in $\mathbb{R}[\lambda, \sigma]$ generated by the equations (8.1) contains elements $a_{12} \lambda_{12}+b_{12}, a_{14} \lambda_{14}+b_{14}, a_{23} \lambda_{23}+b_{23}$ with the $a_{i j}, b_{i j}$ polynomials in $\mathbb{R}[\sigma]$ not vanishing identically on the model, but it does not contain similar elements $a_{41} \lambda_{41}+b_{41}$ or $a_{31} \lambda_{32}+b_{32}$. Furthermore, running a Gröbner basis computation on the fiber equations (8.3) with randomly specialized values incorrectly suggests that the graph has degree of identifiability 3 . These issues disappear when introducing the auxiliary variable $\delta$ and imposing $\delta \cdot \operatorname{det}(I-\Lambda)-1=0$, with $\Sigma$ specialized in the second case. Then the algorithm proves that $G$ is rationally identifiable. In fact, $G$ is HTC-identifiable, because Theorem 1 from the main paper applies with the ordering $2 \prec 3 \prec 1 \prec 4 \prec 5$ and the sets

$$
Y_{2}=\{1,4\}, \quad Y_{3}=\{2\}, \quad Y_{1}=\{3\}, \quad Y_{4}=\{1\}, \quad Y_{5}=\emptyset .
$$

\section{References.}

[1] Thomas H. Cormen, Charles E. Leiserson, Ronald L. Rivest, and Clifford Stein. Introduction to algorithms. MIT Press, Cambridge, MA, second edition, 2001. ISBN 0-262-03293-7.

[2] David Cox, John Little, and Donal O'Shea. Ideals, varieties, and algorithms. Undergraduate Texts in Mathematics. Springer, New York, third edition, 2007. ISBN 978-0-387-35650-1; 0-387-35650-9. An introduction to computational algebraic geometry and commutative algebra.

[3] Wolfram Decker, Gert-Martin Greuel, Gerhard Pfister, and Hans Schönemann. SiNGULAR 3-1-3 - A computer algebra system for polynomial computations, 2011. http://www. singular.uni-kl.de.

[4] Thomas W. Dubé. The structure of polynomial ideals and Gröbner bases. SIAM J. Comput., 19(4):750-773, 1990.

[5] L. R. Ford, Jr. and D. R. Fulkerson. Flows in networks. Princeton University Press, Princeton, N.J., 1962. 
[6] Luis D. Garcia-Puente, Sarah Spielvogel, and Seth Sullivant. Identifying causal effects with computer algebra. In Peter Grünwald and Peter Spirtes, editors, Proceedings of the 26th Conference on Uncertainty in Artificial Intelligence (UAI). AUAI Press, 2010.

[7] Ernst W. Mayr and Albert R. Meyer. The complexity of the word problems for commutative semigroups and polynomial ideals. Adv. Math., 46:305-329, 1982.

DEPARTMENT OF Statistics

The University of ChicAgo

Chichao, IL, U.S.A.

E-MAIL: rina@uchicago.edu drton@uchicago.edu
Department of Mathematics and Computer Science Eindhoven University of TEChNOLOGY

Eindhoven, The Netherlands; AND

Centrum voor Wiskunde en Informatica

Amsterdam, The Netherlands

E-MAIL: j.draisma@tue.nl 